\title{
Large Eddy Simulation of a Lifted Ethylene Flame using a Dynamic Nonequilibrium Model for Subfilter Scalar Variance and Dissipation Rate
}

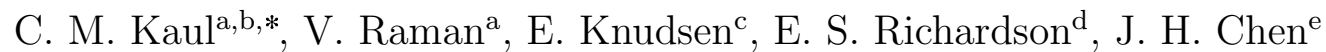 \\ ${ }^{a}$ Dept. of Aerospace Engineering, The University of Texas at Austin, Austin, TX, USA \\ ${ }^{b}$ Center for Turbulence Research, Stanford University, Stanford, CA, USA \\ ${ }^{c}$ Dept. of Mechanical Engineering, Stanford University, Stanford, CA, USA \\ ${ }^{d}$ School of Engineering Sciences, University of Southampton, Southampton, UK \\ ${ }^{e}$ Sandia National Lab, Combustion Research Facility, Livermore, CA, USA
}

\begin{abstract}
Accurate prediction of nonpremixed turbulent combustion using large eddy simulation (LES) requires detailed modeling of the mixing between fuel and oxidizer at scales finer than the LES filter resolution. In conserved scalar combustion models, the small scale mixing process is quantified by two parameters, the subfilter scalar variance and the subfilter scalar dissipation rate. The most commonly used models for these quantities assume a local equilibrium exists between production and dissipation of variance. Such an assumption has limited validity in realistic, technically relevant flow configurations. However, nonequilibrium models for variance and dissipation rate typically contain a model coefficient whose optimal value is unknown a priori for a given simulation. Furthermore, conventional dynamic procedures are not useful for estimating the value of this coefficient. In this work, an alternative dynamic procedure based on the transport equation for subfilter scalar variance is presented, along with a robust conditional averaging approach for evaluation of the
\end{abstract}

\footnotetext{
*Corresponding author. Mailing address: Center for Turbulence Research, Building 500, Stanford University, Stanford, CA 94305-3035

Email address: cmkaul@stanford.edu (C. M. Kaul)
} 
model coefficient. This dynamic nonequilibrium modeling approach is used for simulation of a turbulent lifted ethylene flame, previously studied using DNS by Yoo et al. (Proc. Comb. Inst., 2011). The predictions of the new model are compared to those of a static nonequilibrium modeling approach using an assumed model coefficient, as well as those of the equilibrium modeling appoach. The equilibrium models are found to systematically underpredict both subfilter scalar variance and dissipation rate. Use of the dynamic procedure is shown to increase the accuracy of the nonequilibrium modeling approach. However, numerical errors that arise as a consequence of grid-based implicit filtering appear to degrade the accuracy of all three modeling options. Thus, while these results confirm the usefulness of the new dynamic model, they also show that the quality of subfilter model predictions depends on several factors extrinsic to the formulation of the subfilter model itself.

Keywords: Large eddy simulation, subfilter modeling, dynamic modeling, lifted flame

Word count: 6140, based on Method 2

Form of presentation: Oral

Colloquium: Turbulent Flames 


\section{Introduction}

In large eddy simulation (LES) of turbulent combustion, the description of small scale scalar mixing is crucial for the accurate prediction of turbulence-chemistry interaction [1]. Typically, this mixing is characterized by two variables, namely, scalar variance and scalar dissipation rate. The former quantifies the level of unmixedness at the scales not resolved by LES, and the latter describes the rate of mixing of the scalars that will lead to a decay of the scalar variance. The turbulent length scales are divided into resolved and unresolved (subfilter) scale in LES, with the scalar variance referring to the subfilter scalar distribution. By definition, dissipation rate is proportional to scalar gradient squared, and is active only at the small scales. Consequently, both these quantities are not directly resolved by LES and need to be modeled. It is well established that small errors in these models can significantly alter lift-off heights of flames [2], soot formation and evolution [3], as well as pollutant predictions [4].

While the small-scale mixing of all gas-phase scalars is important, determination of the dissipation rate and variance of a conserved scalar is particularly relevant, given the widespread use of mixture-fraction based models for combustion (including flamelet models [5-7] and conditional moment closure [8-11]). Typically, the models for dissipation rate and variance are related to one another, and utilize a major simplification called the equilibrium assumption. Here, the local production of subfilter variance is assumed to be exactly balanced by scalar dissipation, leading to algebraic models for both quantities. In particular, this assumption allows the use of the so-called dynamic modeling approach $[12,13]$, which enables the computation of model coefficients in situ. Since one of the

main drawbacks of the Reynolds-averaged Navier-Stokes (RANS) simulations is the lack of universal values of model coefficients, this ability in LES to determine coefficient values on 
the fly is considered to be one of the principal reasons for the accuracy of the technique. However, the use of the local equilibrium assumption introduces large errors, and has been found to vastly over predict scalar mixing (and hence scalar dissipation rate).

The focus of this work is to demonstrate a novel nonequilibrium model for variance and dissipation rate, which relies on the solution of a transport equation for subfilter variance. In the past, this approach had been eschewed since it requires the specification of a model coefficient. Here, this hurdle is overcome by formulating a dynamic modeling approach for the entire transport equation. The model is tested using LES of a lifted flame, with both a priori and a posteriori comparisons with a high-resolution direct numerical simulation (DNS) [14].

\section{Subfilter models for scalar mixing}

Combustion modeling approaches in LES are commonly based on the mixture fraction, $Z$, which is a conserved scalar in gas-phase systems [1]. The transport equation for mixture fraction can be written as

$$
\frac{\partial \bar{\rho} \widetilde{Z}}{\partial t}+\frac{\partial \bar{\rho} \tilde{u}_{i} \widetilde{Z}}{\partial x_{i}}=\frac{\partial}{\partial x_{i}}\left[\bar{\rho}\left(\widetilde{D}+D_{T}\right) \frac{\partial \widetilde{Z}}{\partial x_{i}}\right],
$$

where $\bar{\rho}$ is the filtered density, $\widetilde{Z}$ is the Favre-filtered mixture fraction, and $\widetilde{u_{i}}$ is the filtered velocity. The spatial filtering operation $\overline{(\cdot)}$ is characterized by a filterwidth $\Delta$ and is related to the density-weighted Favre filtering operation by $\widetilde{Z}=\overline{\rho Z} / \bar{\rho}$. The remaining quantities in Eq. 1 are $\widetilde{D}$, the diffusivity of the mixture fraction that is typically equated to the thermal diffusivity, and $D_{T}$, a modeled eddy diffusivity.

However, knowledge of the filtered mixture fraction field is insufficient to accurately predict combustion. Models are required for the subfilter scalar unmixedness (or variance) 
as well as the dissipation rate defined as

$$
Z_{v}=\widetilde{Z^{2}}-\widetilde{Z}^{2}
$$

and

$$
\chi_{Z}=2 D \frac{\partial Z}{\partial x_{i}} \frac{\partial Z}{\partial x_{i}}
$$

To obtain the filtered thermochemical composition, these variables are related through a one-point, one-time probability density function (PDF) as follows

$$
P\left(Z, \chi_{Z}\right)=\beta\left(\widetilde{Z}, Z_{v}\right) \delta\left(\chi_{Z}-\widetilde{\chi_{Z}}\right)
$$

where $\beta$ refers to a beta PDF [15], and $\delta$ is a delta PDF. This joint PDF is then convoluted with the flamelet mapping function to obtain the required gas phase composition.

To determine scalar variance, it is possible to construct a variance transport equation (VTE) similar to that used in RANS formulations [16],

$$
\frac{\partial \bar{\rho} Z_{v}}{\partial t}+\frac{\partial \bar{\rho} \tilde{u}_{i} Z_{v}}{\partial x_{i}}=\frac{\partial}{\partial x_{i}}\left[\bar{\rho}\left(\widetilde{D}+D_{T}\right) \frac{\partial Z_{v}}{\partial x_{i}}\right]+2 \bar{\rho} D_{T} \frac{\partial \tilde{Z}}{\partial x_{i}} \frac{\partial \tilde{Z}}{\partial x_{i}}-\bar{\rho} \tilde{\epsilon} Z
$$

where $\tilde{\epsilon}_{Z}$ is the subfilter dissipation rate given by

$$
\tilde{\epsilon}_{Z}=\tilde{\chi}_{Z}-2 \widetilde{D} \frac{\partial \widetilde{Z}}{\partial x_{i}} \frac{\partial \widetilde{Z}}{\partial x_{i}}
$$

and appears as an unclosed sink term. In terms of modeling, both $Z_{v}$ and $\tilde{\chi}_{Z}$ (or $\epsilon_{Z}$ ) need to be modeled, and are related by the transport of scalar variance.

In the equilibrium modeling approach, a local balance is assumed to exist between the rate at which variance is destroyed at the small scales and the rate at which it is produced, 
as given by the second term on the right hand side of Eq. 5. This results in the model

$$
\tilde{\epsilon}_{Z}=2 D_{T} \frac{\partial \widetilde{Z}}{\partial x_{i}} \frac{\partial \widetilde{Z}}{\partial x_{i}}
$$

for the subfilter dissipation $[13,17]$. The corresponding variance model is

$$
Z_{v}=\mathcal{C}_{v} \Delta^{2} \frac{\partial \widetilde{Z}}{\partial x_{i}} \frac{\partial \widetilde{Z}}{\partial x_{i}}
$$

The coefficient $\mathcal{C}_{v}$ in Eq. 8 can be determined dynamically $[13,18]$. Dynamic modeling is a procedure specific to LES that allows model coefficients to be estimated from the filtered fields during a simulation, rather than prescribed a priori $[19,20]$.

Prior studies have shown that the equilibrium approach is invalid even in the simplest of flows [21, 22], leading to large underprediction of scalar variance. Consequently, the use of the VTE (Eq. 5) is a viable alternative. This transport equation can also be written in an alternate form based on the second moment of the scalar,

$$
\frac{\partial \bar{\rho} \widetilde{Z^{2}}}{\partial t}+\frac{\partial \bar{\rho} \tilde{u}_{i} \widetilde{Z^{2}}}{\partial x_{i}}=\frac{\partial}{\partial x_{i}}\left[\bar{\rho}\left(\widetilde{D}+D_{T}\right) \frac{\partial \widetilde{Z^{2}}}{\partial x_{i}}\right]-\bar{\rho} \widetilde{\chi} Z
$$

with the variance then be computed from its definition (Eq. 2). It has been shown that the STE provides a numerically accurate formulation [21]. Here, both VTE and STE formulations will considered in order to determine the role of numerical errors in the model predictions.

Since the equilibrium model (Eq. 7) can no longer be used with a transport equationbased variance description, the dissipation rate is typically closed using

$$
\tilde{\epsilon}_{Z}=\mathcal{C}_{\tau} \frac{Z_{v}}{\tau_{Z}}
$$


where $\mathcal{C}_{\tau}$ is a model coefficient and $\tau_{Z}$ is a mixing timescale [16]. Essentially, this model relates the small-scale dissipation rate to the timescales associated with filter-scale turbulent structures. A variety of mixing time scale expressions can be formulated. Here, the common closure

$$
\tau_{Z}=\frac{\Delta^{2}}{\widetilde{D}+D_{T}}
$$

is used $[4,23,24]$.

The model coefficient, $\mathcal{C}_{\tau}$, is dependent on the filterwidth as well as the local distribution of length scales. Since no universal value is applicable, a wide range of values have been used in the past $[4,24]$. A higher accuracy could be gained if the model coefficient could be evaluated dynamically. With this in mind, the VTE-based dynamic modeling approach is formulated next.

\section{VTE-based dynamic model}

\subsection{Basic Formulation}

The new dynamic scalar dissipation rate and variance model utilizes a scale similarity assumption to formulate a scaling law. Consider $\Delta$ to be the filterwidth, and the test filter $\widehat{\Delta}$ to be of size $2 \Delta$. The spatial test filtering operation is indicated by $\widehat{(\cdot)}$ and can be used to form a test filter scale Favre filtering operator $\widetilde{(\cdot)}$ that is related to the LES filter scale Favre filter by $\breve{Z}=\widehat{\overline{\rho Z}} / \hat{\bar{\rho}}=\widehat{\bar{\rho} \widetilde{Z}} / \hat{\bar{\rho}}$. Using this notation, the transport equation for the sub-test filter scalar variance, given by $Z_{t}=\widetilde{Z Z}-\check{Z} \breve{Z}$, can be written as

$$
\frac{\partial \hat{\bar{\rho}} Z_{t}}{\partial t}+\frac{\partial \hat{\bar{\rho}} \breve{u}_{i} Z_{t}}{\partial x_{i}}=\frac{\partial}{\partial x_{i}}\left[\hat{\bar{\rho}}\left(D+D_{T}^{t}\right) \frac{\partial Z_{t}}{\partial x_{i}}\right]+2 \hat{\bar{\rho}} D_{T}^{t} \frac{\partial \check{Z}}{\partial x_{i}} \frac{\partial \check{Z}}{\partial x_{i}}-\mathcal{C}_{\tau} \hat{\bar{\rho}} \frac{Z_{t}}{\tau_{Z}^{t}}
$$

A model quantity that is evaluated at the test filter level is indicated by a superscript $t$

(e.g. $D_{T}^{t}$ ) to distinguish it from a subfilter model quantity which undergoes test filtering 
(e.g. $\widehat{D_{T}}$ ). Note that $\mathcal{C}_{\tau}$ is assumed to have the same value at both filter scales. Test filtering Eq. 5 in its entirety and subtracting it from Eq. 12 gives the relationship

$$
\mathcal{C}_{\tau} X=Y=F+P-T
$$

if variations in $\mathcal{C}_{\tau}$ values over length scales smaller than $\widehat{\Delta}$ are neglected. The quantity $X$ on the left hand side of Eq. 13 is given by

$$
X=\hat{\bar{\rho}} \frac{Z_{t}}{\tau_{Z}^{t}}-\widehat{\left(\bar{\rho} \frac{Z_{v}}{\tau_{Z}}\right)}
$$

The first two terms on the right hand side of Eq. 13 represent the differences in convective and diffusive fluxes of variance,

$$
F=\frac{\partial}{\partial x_{i}}\left[\widehat{\bar{\rho} Z_{v} \widetilde{u_{i}}}-\bar{\rho}\left(D+\widetilde{D_{T}}\right) \frac{\partial Z_{v}}{\partial x_{i}}\right]-\frac{\partial}{\partial x_{i}}\left[\hat{\bar{\rho}} Z_{t} \breve{u_{i}}+\hat{\bar{\rho}}\left(D+D_{T}^{t}\right) \frac{\partial Z_{t}}{\partial x_{i}}\right]
$$

and in production of variance,

$$
P=2 \hat{\bar{\rho}} D_{T}^{t} \frac{\partial \breve{Z}}{\partial x_{i}} \frac{\partial \breve{Z}}{\partial x_{i}}-2 \bar{\rho} D_{T} \frac{\partial \widetilde{Z}}{\partial x_{i}} \frac{\partial \widetilde{Z}}{\partial x_{i}}
$$

at the test and LES filter scale. The third term represents accumulation or loss of variance between LES filter and test filter scales,

$$
T=\frac{\partial \hat{\bar{\rho}} L_{v}}{\partial t}
$$

where $L_{v}$ is the variance Leonard term given by $L_{v}=\widetilde{\widetilde{Z} \widetilde{Z}}-\widetilde{\widetilde{Z}} \widetilde{Z}$ or, equivalently, by $L_{v}=Z_{t}-\bar{Z}_{v}$.

Dynamic model coefficients are typically evaluated using one of a variety of averaging 
procedures to reduce numerically destabilizing fluctuations in the coefficient values. Additionally, the use of an averaging procedure reflects the inherently statistical nature of LES subfilter modeling and can incorporate an ordinary least squares regression technique $[13,25]$. A priori analysis of the model $[22]$ in the framework of the optimal estimator concept $[18,26,27]$ suggests a novel conditional averaging procedure for the determination of $\mathcal{C}_{\tau}$. Three-dimensional, spatially and temporally varying fields of $\mathcal{C}_{\tau}$ values are found from

$$
\mathcal{C}_{\tau}(\phi)=\frac{\left\langle X Y \mid \phi=\bar{\rho} Z_{v} / \tau_{Z}, X Y>0\right\rangle}{\left\langle X^{2} \mid \phi=\bar{\rho} Z_{v} / \tau_{Z}, X Y>0\right\rangle} .
$$

Conceptually, this approach seeks to determine $\mathcal{C}_{\tau}$ by averaging over instantaneous flow structures. Therefore, the average can be evaluated by sampling over the entire computational domain, with the spatial variation of the conditioning variable substituting for any explicit geometrical dependence. Further details of the coefficient determination procedure will be provided in Sec. 4.3, in the context of the flow configuration, combustion modeling approach, and numerical implementation used to carry out a posteriori evaluation of the model.

\section{Simulation Description}

The LES test case chosen for evaluation of the new model is a lifted ethylene jet flame, previously studied using DNS [14]. The availability of a DNS data set for comparison facilitates direct assessment of the scalar dissipation rate and scalar variance model predictions, in contrast to an indirect assessment based on the predicted flow thermochemistry.

\subsection{Flow Configuration and DNS Parameters}

The flow configuration consists of a three-dimensional slot burner [14]. Its primary characteristics are summarized here, with additional details available in Ref. 14 . 
The central jet, of width $H=2 \mathrm{~mm}$ has a chemical composition of $18 \%$ ethylene $\left(\mathrm{C}_{2} \mathrm{H}_{4}\right)$ and $82 \%$ nitrogen by volume with a temperature of $550 \mathrm{~K}$. The coflow is composed of air, heated to $1550 \mathrm{~K}$ and at atmospheric pressure. The combustion chemistry is described by a 22 species reduced ethylene mechanism [28]. The mean velocities of the central jet and coflow are $204 \mathrm{~m} / \mathrm{s}$ and $20 \mathrm{~m} / \mathrm{s}$, respectively. Velocity fluctuations from an auxiliary simulation of homogeneous, isotropic turbulence are added to the mean inlet velocity, imparting a $10 \%$ turbulence intensity to the central jet. The Reynolds number of the flow, based on the central jet width and mean velocity, is 10,000 .

The computation domain spans $L_{x}=15 H$ in the streamwise direction, $L_{y}=20 H$ in the stream normal direction, and $L_{z}=3 H$ in the periodic spanwise direction. The computational mesh of the DNS is uniform over the region of the domain containing the flame with a grid spacing of $15 \mu \mathrm{m}$. The simulation was performed using a fourth order Runge-Kutta method for time integration and an eighth order central difference scheme for spatial discretization.

\subsection{Combustion Model}

In the LES computations described in this work, combustion is modeled using an unsteady flamelet/progress variable approach [29], accounting for differential diffusion effects $[30,31]$. The flamelet solutions are calculated using the same 22 species ethylene mechanism employed in the DNS [28]. The progress variable, $C$, is defined as the sum of the mass fractions of $\mathrm{H}_{2} \mathrm{O}, \mathrm{H}_{2}, \mathrm{CO}$, and $\mathrm{CO}_{2}$. The value of a filtered thermochemical variable $\widetilde{\psi}$ is accessed from the unsteady flamelet library $\mathcal{F}$ according to

$$
\widetilde{\psi}=\mathcal{F}\left(\widetilde{Z}, Z_{v}, \widetilde{C}, \tilde{\chi}\right)
$$




\subsection{LES Numerical Parameters and Implementation}

The LES computations were performed on the DNS computational domain, using filtered inflow boundary conditions. The LES mesh consists of $171 \times 172 \times 32$ points in the three coordinate directions. A structured LES solver with second-order discretization schemes for the nonlinear and viscous terms were used [32]. The nonlinear terms in the scalar equations were treated using the BQUICK scheme [33]. A semi-implicit temporal integration was used [32]. Standard dynamic models were used to determine eddy viscosity and eddy diffusivity values [12].

The combustion model's predictions of density are sensitive to the reference stoichiometric scalar dissipation rate value. This quantity depends on the local value of $\tilde{\chi}_{Z}$, which in turn depends on the subfilter dissipation rate model coefficient $\mathcal{C}_{\tau}$. Therefore, the dynamic estimate for $\mathcal{C}_{\tau}$ must be made in way that avoids potentially numerically destabilizing fluctuations in density. The conditional coefficient evaluation procedure (Eq. 18) naturally satisfies this requirement in statistically stationary flow such as the one considered here, because the conditional average is also, implicitly, a temporal average. At the outset of a simulation, when relatively few samples have been accumulated, sudden variations in $\mathcal{C}_{\tau}$ can still occur. Additionally, the time derivative term $T$ of the dynamic model (Eq. 17) must typically be computed as a backwards difference. Of course, this is not possible at the first timestep.

To deal with both these issues, the dynamic estimate of $\mathcal{C}_{\tau}$ was computed as follows. On the first timestep of a simulation, $\bar{\rho} L_{v}$ is calculated from the initial conditions and $\mathcal{C}_{\tau}$ is set to some pre-selected fixed value, here $\mathcal{C}_{\tau}=2$. On the second and subsequent timesteps, $T$ is computed from the previous and current timestep values of $\bar{\rho} L_{v}$. The other terms of the model (Eqs. 14-16) can be found from the most recent velocity and scalar fields. Next, the quantities $X Y(\mathbf{x}, t)$ and $X^{2}(\mathbf{x}, t)$ are grouped into discrete bins (indexed by $p$ ) based on 
the value of the conditioning variable $\phi(\mathbf{x}, t)$ and satisfaction of the condition $X Y(\mathbf{x}, t)>0$. A running average $X Y_{p}$ and $X_{p}^{2}$ is kept for each bin, and $\mathcal{C}_{\tau, p}$ is found at the bin midpoint value $\phi_{p}$ from the updated $X Y_{p}$ and $X_{p}^{2}$ averages. To return to the physical computational mesh, $\mathcal{C}_{\tau}(\mathbf{x}, t)$ is determined by interpolation between $\mathcal{C}_{\tau, p}$ values. A decaying exponential weighting function is used to transition between the pre-selected coefficient value and the dynamically determined one. A transition time equal to approximately one third of a jet timescale, $\tau_{j}=L_{x} / U_{j}$, was found to ensure sufficient smoothness.

\subsection{A Posteriori Comparison Issues}

Performing effective and informative a posteriori model evaluations under current LES simulation methods is not a simple endeavor. Undoubtedly, the most straightforward way to find out how a model performs in a practical LES simulation is simply to carry out the simulation using the model. However, model assessments made in this way may lack generalizability, since they also include the effects of all the other approximations, physical and numerical, specific to that simulation. The use of implicit, grid-based filtering in LES causes a close coupling of numerical and modeling errors since both predominantly affect length scales of the flow close to the grid resolution and nominal LES filterwidth. Three factors affecting LES of reacting flow compound this problem. First, the dependence of the large scale flow dynamics on the small scales in LES of combustion is stronger than that encountered in LES of nonreactive flow [1]. Second, the response of the combustion model to errors in its input parameters can be strongly nonlinear. Third, second-order spatial discretizations are widely used in LES of combustion despite their high truncation error due to their greater numerical robustness [32].

In order to compare the LES model predictions to the DNS results, the DNS data fields must be filtered. Since the LES mesh is non-uniform and hence has a variable filterwidth, 
the use of a uniform filterwidth to filter the DNS data might introduce errors. To test the impact of these differences, the DNS data was filtered using two filter sizes set of 7 and 9 DNS grid spacings. It was found that the difference between these two filtered fields were much smaller than the difference between the LES results and the filtered DNS field. Hence, it was concluded that the filter definition used for processing the DNS data was not a major source of errors. The filtered DNS results plotted in Sec. 5 were computed with $\Delta=135 \mu \mathrm{m}$.

\section{Results}

In this section, three sets of scalar variance and dissipation rate modeling results are compared to DNS values. The three cases are a dynamic nonequilibrium modeling approach combining the STE with dynamic estimation of $\mathcal{C}_{\tau}$, a static nonequilibrium modeling approach using the STE and an assumed coefficient value $\mathcal{C}_{\tau}=2$, and the equilibrium modeling approach. The coefficient $\mathcal{C}_{v}$ in the equilibrium variance model (Eq. 8) is determined from the Leonard term expansion dynamic model [18]. Fig. 1 shows instantaneous contours of temperature from DNS and from LES using the dynamic nonequilibrium modeling approach.

Time averaged profiles of $\widetilde{\chi_{Z}}, Z_{v}$, and related quantities are shown at axial locations of $x / H=0.5,3$, and 6 , which is approximately equal to the average flame lift-off height of $x / H=5.8$ found in the DNS. This range of axial locations was selected for three reasons. First, accurate modeling of the scalar dissipation rate in this region of the flow is highly relevant for capturing the flame base dynamics in LES. Second, the flow dynamics at these locations are minimally affected by heat release. This reduces feedback from errors intrinsic to the unsteady flamelet combustion model to the scalar variance and dissipation rate predictions that are the main focus of the analysis. Third, as the distance from the 

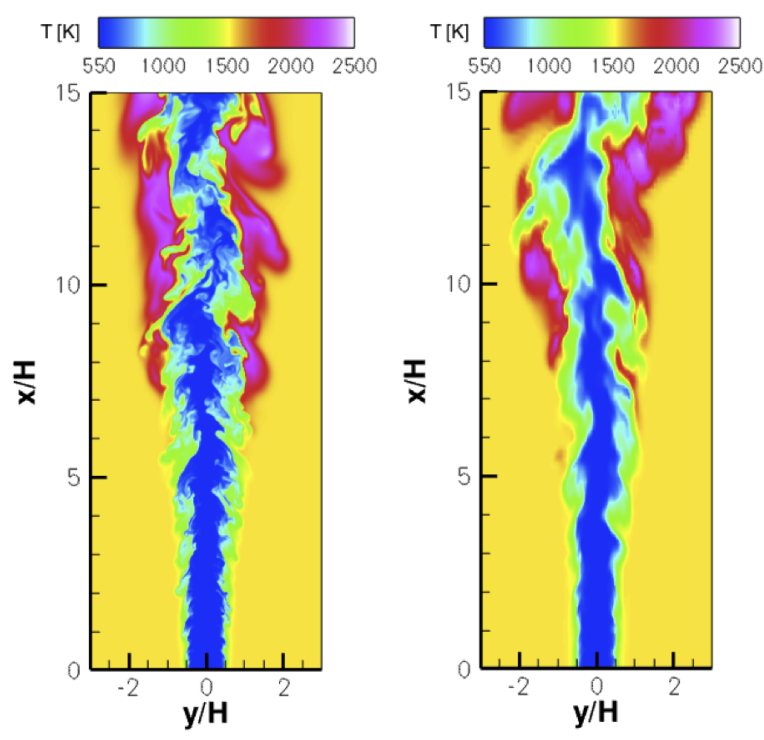

Figure 1: Representative instantaneous snapshots of temperature in (left) DNS; (right) LES, using the dynamic nonequilibrium model.

inlet increases, all the sources of error mentioned in Sec. 4.4 potentially accumulate and impact scalar modeling outcomes. Thus, it becomes increasingly difficult to isolate the effect of the modeling closure.

\subsection{Scalar Dissipation Rate Modeling}

Fig. 2 shows time averaged cross stream profiles of the filtered scalar dissipation rate at three axial locations up to $x / H=6$. At each of these locations, the dynamic nonequilibrium modeling approach predicts the highest values of $\tilde{\chi}_{Z}$, followed by the static nonequilibrium approach. This is directly attributable to the improved estimate of $\mathcal{C}_{\tau}$ achieved with the dynamic model. Values of $\mathcal{C}_{\tau}$ obtained from the dynamic model ranged between just over 13 at low values of the conditioning variable $\phi=\bar{\rho} Z_{v} / \tau_{Z}$ to just under 3 at the highest, and most rarely encountered, values of $\phi$. An instantaneous snapshot of $\mathcal{C}_{\tau}$ values is shown in Fig. 3. 


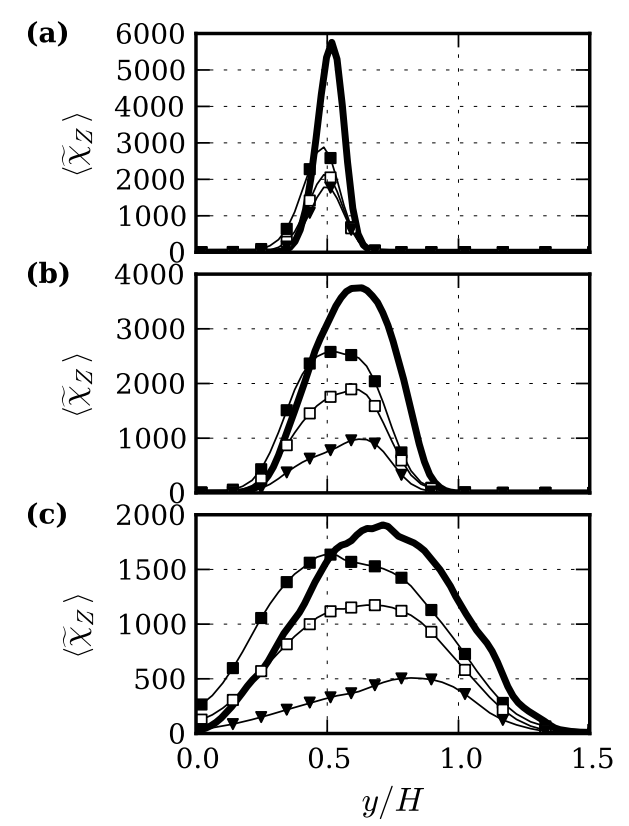

Figure 2: Time-averaged cross stream profiles of filtered scalar dissipation rate $\left\langle\tilde{\chi}_{Z}\right\rangle$ at axial locations (a) $x / H=0.5$ (b) $x / H=3$ (c) $x / H=6$ from (一) filtered DNS; (black squares) LES, dynamic $\mathcal{C}_{\tau}$; (white squares) LES, $\mathcal{C}_{\tau}=2$; (black triangles) LES, equilibrium model.

The equilibrium modeling approach yields substantially lower values of $\tilde{\chi}_{Z}$ than either of the nonequilibrium modeling cases. Computation of the equilibrium model with a higher order finite difference scheme confirmed that part, but not all, of its underprediction is numerical in origin. The trends shown in Fig. 2 were found to persist in profiles obtained farther downstream (not shown). It is notable that the $\tilde{\chi}_{Z}$ profiles in Fig. 2 resulting from the nonequilibrium modeling approaches (both static and dynamic) are offset from the DNS profiles, peaking closer to the core of the jet. However, if this offset is corrected for, the dynamic nonequilibrium model generally gives the best match to the DNS.

The offset of the nonequilibrium models' filtered scalar dissipation rate profiles from the DNS profiles is an interesting feature of these results. Since it is observable for both 


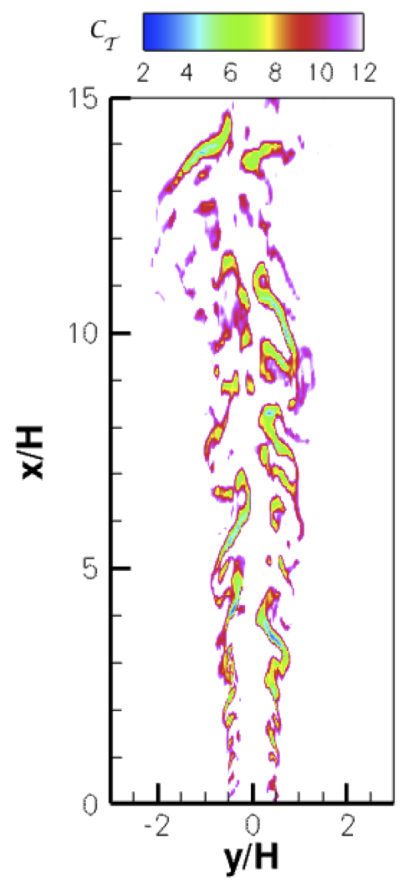

Figure 3: Representative instantaneous snapshot of $\mathcal{C}_{\tau}$ values predicted by the dynamic model.

dynamic and static approaches, it is not induced by the spatial variation of the $\mathcal{C}_{\tau}$ values obtained from the dynamic procedure. Instead, it appears to be related to some other feature of the model. More detailed discussion of this phenomenon is deferred to Sec. 5.2. As discussed previously, the filtered scalar dissipation rate can be separated into a resolved component $\chi_{Z \text {,res }}$, for which no modeling is required, and a subfilter component $\tilde{\epsilon}_{Z}$, which must be modeled. Only $\tilde{\epsilon}_{Z}$ is directly affected by the dynamic estimate of $\mathcal{C}_{\tau}$. The resolved and subfilter components of the dissipation rate obtained from the DNS data and from the dynamic nonequilibrium model are illustrated in Fig. 4. The axial locations shown correspond to those in Fig. 2. Nearest the inlet [Fig. 4(a)], the DNS filtered dissipation rate is dominated by its resolved component. Moving downstream, $\chi_{Z \text {,res }}$ continues to make up a large portion of the filtered dissipation rate, $\tilde{\chi}_{Z}$. The LES results show far 


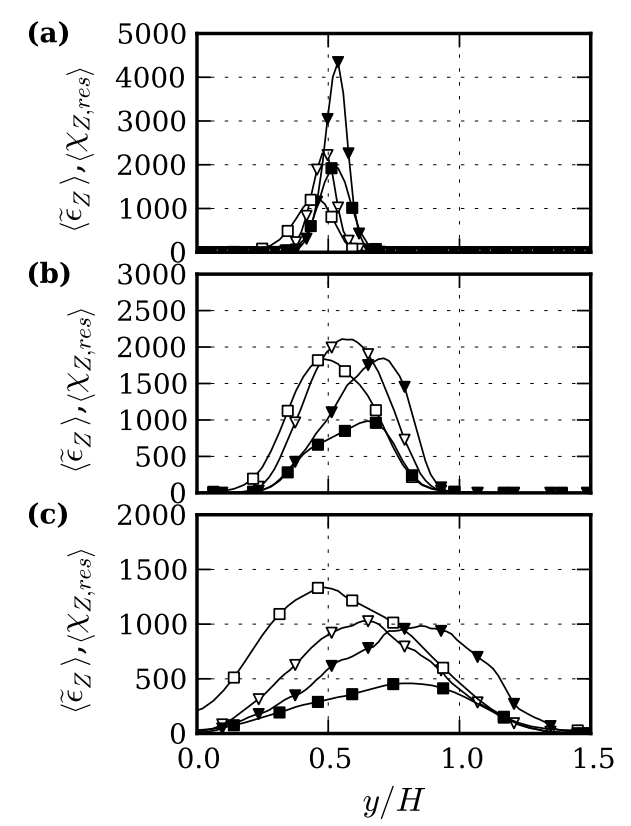

Figure 4: Time-averaged cross stream profiles of subfilter scalar dissipation rate $\left\langle\widetilde{\epsilon}_{Z}\right\rangle$ and resolved scalar dissipation $\left\langle\chi_{z, \text { res }}\right\rangle$ at axial locations (a) $x / H=0.5$ (b) $x / H=3$ (c) $x / H=6$. Results are shown for (white triangles) exact subfilter dissipation from DNS; (black triangles) resolved dissipation from DNS; (white squares) modeled subfilter dissipation from LES, using dynamic $\mathcal{C}_{\tau}$; (black squares) resolved subfilter dissipation from LES using the dynamic nonequilibrium model. 
lower values of $\chi_{Z \text {,res }}$, causing much of the underprediction of peak $\tilde{\chi}_{Z}$ values. Like the equilibrium model for $\tilde{\chi}_{Z}$, the resolved dissipation depends on the square of the magnitude of the filtered scalar gradient and is prone to under prediction in LES computations using grid-based filtering $[21,34]$. It was verified that increasing the order of the finite difference scheme used to compute $\chi_{Z \text {,res }}$ increased that quantity's magnitude.

\subsection{Scalar Variance Modeling}

The values of subfilter dissipation predicted using Eq. 10 in the nonequilibrium modeling approach depend directly on the subfilter scalar variance values. The close relationship between the two quantities is evident in a comparison of Fig. 4 to Fig. 5, which shows the subfilter variance predictions of the three modeling cases as well as the DNS results. All three models underpredict the variance near the inlet. Farther downstream, the nonequilibrium models yield variance values that are too large, while the equilibrium model continues to underpredict the variance.

The inward offset of the $\tilde{\epsilon}_{Z}$ profiles of the nonequilibrium model is clearly due to a similar offset of the $Z_{v}$ profiles. This error in the variance cannot be directly attributed to dissipation rate modeling errors. Instead, it must be due to terms in the variance model related to the transport and production of variance. In Sec. 2, it was mentioned that the STE lacks an explicit variance production term. However, an effective variance production term is contained within the model formulation. The evolution of the variance under the VTE model can be written as

$$
\frac{d \bar{\rho} Z_{v, \mathrm{VTE}}}{d t}=\frac{d \bar{\rho} \widetilde{Z^{2}}}{d t}-\frac{d \bar{\rho} \widetilde{Z}^{2}}{d t}
$$




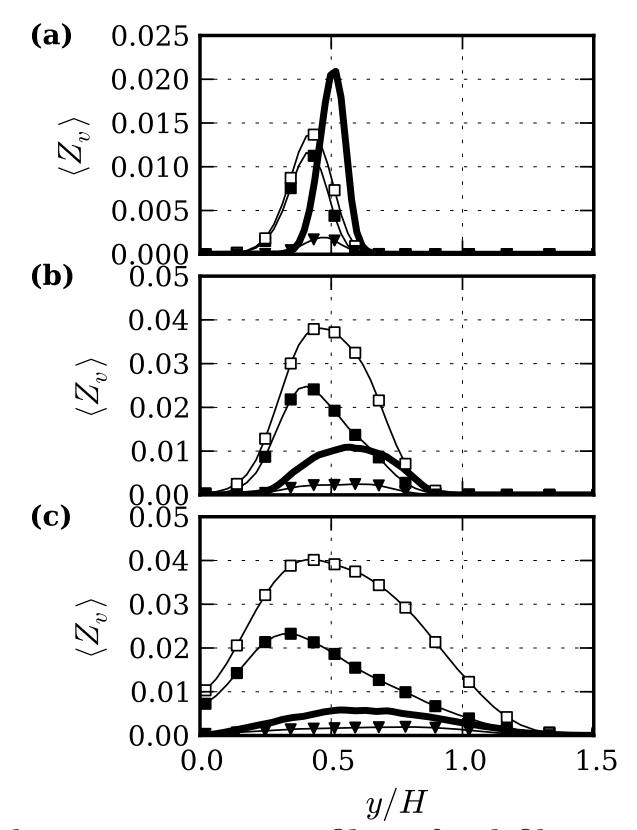

Figure 5: Time-averaged cross stream profiles of subfilter scalar variance $Z_{v}$ at axial locations (a) $x / H=0.5$ (b) $x / H=3$ (c) $x / H=6$ from (一) filtered DNS; (black squares) LES, dynamic $\mathcal{C}_{\tau}$; (white squares) LES, $\mathcal{C}_{\tau}=2$; (black triangles) LES, equilibrium model.

In contrast, the evolution of the variance under the STE can be thought of as

$$
\frac{d \bar{\rho} Z_{v, \mathrm{STE}}}{d t}=\frac{d \bar{\rho} \widetilde{Z^{2}}}{d t}-2 \widetilde{Z} \frac{d \bar{\rho} \widetilde{Z}}{d t} .
$$

In the VTE, variance production appears in the equation for the evolution of $\widetilde{Z}$, the second term on the right hand side of Eq. 20. Comparing this with the second term on the right hand side of Eq. 21 allows the implicit production in the STE to be defined as

$$
\mathcal{P}_{\mathrm{STE}}=\frac{\partial}{\partial x_{i}}\left[\bar{\rho} D_{T} \frac{\partial \widetilde{Z}^{2}}{\partial x_{i}}\right]-2 \widetilde{Z} \frac{\partial}{\partial x_{i}}\left[\bar{\rho} D_{T} \frac{\partial \widetilde{Z}}{\partial x_{i}}\right]
$$


Recall that the explicit production term in the VTE is

$$
\mathcal{P}=2 \bar{\rho} D_{T} \frac{\partial \widetilde{Z}}{\partial x_{i}} \frac{\partial \widetilde{Z}}{\partial x_{i}} .
$$

At the level of continuous equations, $\mathcal{P}_{\mathrm{STE}}$ and $\mathcal{P}$ are equivalent. However, they yield different values when evaluated discretely, as shown in Fig. 6. The disparity between these two variations of the production term is indicative of the sensitivity of LES solutions to the precise form of the equations used. The results shown in Fig. 6 also help to explain the excess variance generated towards the center of jet. Note that these results should not be interpreted as implying that the VTE is more accurate than the STE. Indeed, the greater smoothness of the averaged VTE production term suggest a tendency to lose higher wavenumber fluctuations due to the gradient-squared form of $\mathcal{P}$.

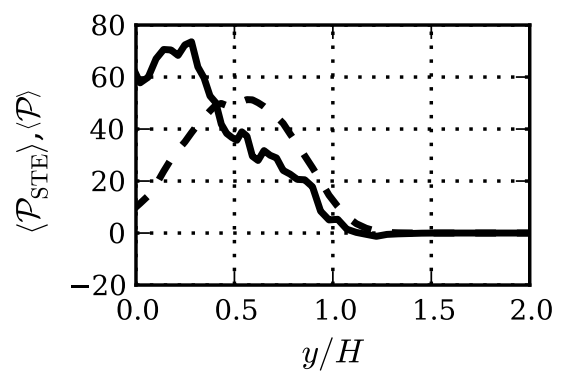

Figure 6: Time-averaged cross stream profiles of production terms at $x / H=6$ from $(-) \mathcal{P}_{\mathrm{STE}} ;(---) \mathcal{P}$.

\section{Summary and conclusions}

In this work, a dynamic nonequilibrium approach for modeling of subfilter scalar variance and dissipation rate in LES has been formulated and used for simulation of a turbulent lifted ethylene flame. The dynamic procedure is based on the variance transport equation and allows optimal values of a flow- and filterwidth-depedent coefficient $\mathcal{C}_{\tau}$ appearing in 
the subfilter dissipation rate closure to be estimated on the fly. Conventional equilibrium and static nonequilibrium modeling approaches are also tested. In the lifted flame simulation, equilibrium modeling approaches are found to substantially underpredict subfilter scalar variance and filtered scalar dissipation rate. In contrast, the dynamic nonequilibrium modeling approach predicts the magnitude of the filtered scalar dissipation rate more accurately while overpredicting the subfilter scalar variance. Comparison of the results of the dynamic and static nonequilibrium modeling approaches shows that this error is not solely attributable to the subfilter dissipation rate closure. Instead, it also reflects errors in the computation of other terms of the model, which are at least partially numerical in nature.

In closing, these simulations confirm the viability of the dynamic nonequilibrium modeling approach for realistic LES computations. However, they also show that grid-based implicit filtering, in addition to negatively affecting the accuracy of simulations, limits the strength of the conclusions that can be drawn from a posteriori analysis due to the close coupling of modeling and numerical errors. Thus, methods to reduce numerical error while maintaining numerical stability are needed in reacting flow LES along with improved subfilter closures, such as the dynamic nonequilibrium modeling approach presented here.

\section{Acknowledgments}

The authors gratefully acknowledge financial support from NASA through grants NNX07AB92A and NNX08AB41A and the HPC resources provided by the High Performance Computing Center at Stanford University that contributed to the research results reported within this paper. 


\section{References}

[1] H. Pitsch, Annual Review of Fluid Mechanics 38 (2006) 453-482.

[2] E. Knudsen, E. S. Richardson, E. M. Doran, H. Pitsch, J. H. Chen, Physics of Fluids (In Revision).

[3] M. E. Mueller, H. Pitsch, Combustion and Flame (In Revision).

[4] V. Raman, H. Pitsch, Proceedings of the Combustion Institute 31 (2006) 1711-1719.

[5] H. Pitsch, H. Steiner, Physics of Fluids 12 (2000) 2541-2554.

[6] V. Raman, H. Pitsch, Combustion and Flame 142 (2005) 329-347.

[7] A. Kempf, R. P. Lindstedt, J. Janicka, Combustion and Flame 144 (2006) 170-189.

[8] R. W. Bilger, Physics of Fluids A: Fluid Dynamics 5 (1993) 436-444.

[9] W. K. Bushe, H. Steiner, Physics of Fluids 11 (1999) 1896-1906.

[10] E. Mastorakos, R. W. Bilger, Physics of Fluids 10 (1998) 1246-1248.

[11] A. Y. Klimenko, R. W. Bilger, Progress in Energy and Combustion Science 25 (1999) $595-687$.

[12] P. Moin, K. Squires, W. Cabot, S. Lee, Physics of Fluids A 3 (1991) 2746-2757.

[13] C. D. Pierce, P. Moin, Physics of Fluids 10 (1998) 3041-3044.

[14] C. S. Yoo, E. S. Richardson, R. Sankaran, J. H. Chen, Proceedings of the Combustion Institute 33 (2011) 1619-1627. 
[15] J. Jimenez, A. Linan, M. M. Rogers, F. J. Higuera, Journal of Fluid Mechanics 349 (1997) 149-171.

[16] N. Peters, Turbulent Combustion, Cambridge University Press, 2000.

[17] S. S. Girimaji, Y. Zhou, Physics of Fluids 8 (1996) 1224-1236.

[18] G. Balarac, H. Pitsch, V. Raman, Physics of Fluids 20 (2008) 035114.

[19] M. Germano, U. Piomelli, P. Moin, W. H. Cabot, Physics of Fluids 7 (1991) 17601765 .

[20] C. Meneveau, J. Katz, Annual Review of Fluid Mechanics 32 (2000).

[21] C. M. Kaul, V. Raman, Physics of Fluids 23 (2011) 035102.

[22] C. M. Kaul, V. Raman, Physics of Fluids (Submitted).

[23] P. J. Colucci, F. A. Jaberi, P. Givi, Physics of Fluids 10 (1998) 499-515.

[24] F. A. Jaberi, P. J. Colucci, S. James, P. Givi, S. B. Pope, Journal of Fluid Mechanics 401 (1999) 85-121.

[25] D. K. Lilly, Physics of Fluids A 4 (1992) 633-635.

[26] A. Moreau, O. Teytaud, J. P. Bertoglio, Physics of Fluids 18 (2006) 105101.

[27] G. Balarac, H. Pitsch, V. Raman, Physics of Fluids 20 (2008) 091701.

[28] Z. Luo, C. S. Yoo, E. S. Richardson, J. H. Chen, C. K. Law, T. Lu, Combustion and Flame 159 (2012) 265-274.

[29] M. Ihme, Y. C. See, Combustion and Flame 157 (2010) 1850-1862. 
[30] H. Pitsch, N. Peters, Combustion and Flame 144 (1998) 26-40.

[31] H. Pitsch, Combustion and Flame 123 (2000) 358-374.

[32] O. Desjardins, G. Blanquart, G. Balarac, H. Pitsch, Journal of Computational Physics 227 (2008) 7125-7159.

[33] M. Herrmann, G. Blanquart, V. Raman, AIAA Journal 44 (2006) 2879-2880.

[34] C. M. Kaul, V. Raman, G. Balarac, H. Pitsch, Physics of Fluids 21 (2009) 055102. 


\section{List of Figure Captions}

Figure 1: Representative instantaneous snapshots of temperature in (left) DNS; (right) LES, using the dynamic nonequilibrium model.

Figure 2: Time-averaged cross stream profiles of filtered scalar dissipation rate $\left\langle\tilde{\chi}_{Z}\right\rangle$ at axial locations (a) $x / H=0.5$ (b) $x / H=3$ (c) $x / H=6$ from (一) filtered DNS; (black squares) LES, dynamic $\mathcal{C}_{\tau}$; (white squares) LES, $\mathcal{C}_{\tau}=2$; (black triangles) LES, equilibrium model.

Figure 3: Representative instantaneous snapshot of $\mathcal{C}_{\tau}$ values predicted by the dynamic model.

Figure 4: Time-averaged cross stream profiles of subfilter scalar dissipation rate $\left\langle\tilde{\epsilon}_{Z}\right\rangle$ and resolved scalar dissipation $\left\langle\chi_{Z, \text { res }}\right\rangle$ at axial locations (a) $x / H=0.5$ (b) $x / H=3$ (c) $x / H=6$. Results are shown for (white triangles) exact subfilter dissipation from DNS; (black triangles) resolved dissipation from DNS; (white squares) modeled subfilter dissipation from LES, using dynamic $\mathcal{C}_{\tau}$; (black squares) resolved subfilter dissipation from LES using the dynamic nonequilibrium model.

Figure 5: Time-averaged cross stream profiles of subfilter scalar variance $Z_{v}$ at axial locations (a) $x / H=0.5$ (b) $x / H=3$ (c) $x / H=6$ from (一) filtered DNS; (black squares) LES, dynamic $\mathcal{C}_{\tau}$; (white squares) LES, $\mathcal{C}_{\tau}=2$; (black triangles) LES, equilibrium model.

Figure 6: Time-averaged cross stream profiles of production terms at $x / H=6$ from $(-)$ $\mathcal{P}_{\mathrm{STE}} ;(--\boldsymbol{)}) \mathcal{P}$. 